\title{
Avaliação do efeito da glicosamina e condroitina na consolidação de fratura: estudo experimental em ratos"
}

\author{
Evaluating the effects of glucosamine and chondroitin \\ in bone healing: experimental study in rats \\ Roberto Guarniero', Éden Dal Molin², Carlos Eduardo Sanches Vaz ${ }^{3}$, \\ Paulo José de Santana ${ }^{4}$, Fernando Takao Cinagawa ${ }^{5}$, William Shimada Tatibana ${ }^{6}$
}

\section{RESUMO}

Objetivo: Avaliar o efeito da administração da condroitina e da glicosamina na consolidação de fratura em modelo animal. Métodos: Este estudo experimental envolveu a utilização de $\mathbf{4 0}$ ratos machos adultos da raça Lewis. Os animais foram randomicamente divididos em quatro grupos de 10 animais cada, assim constituídos: grupo I, com administração de glicosamina; grupo II, com administração de condroitina; grupo III, administração da associação de glicosamina e condroitina; grupo IV, administra-

* Trabalho realizado na Faculdade de Medicina da Universidade Estadual de Londrina - UEL - Londrina (PR), Brasil.

1. Professor Associado Livre-docente da Faculdade de Medicina da Universidade de São Paulo - USP - São Paulo (SP), Brasil.

2. Doutor, Professor Adjunto da Faculdade de Medicina da Universidade Estadual de Londrina - UEL - Londrina (PR), Brasil.

3. Doutor, Professor Adjunto da Faculdade de Medicina da Universidade Estadual de Londrina - UEL - Londrina (PR), Brasil.

4. Doutor, Professor Adjunto da Faculdade de Medicina da Universidade Estadual de Londrina - UEL - Londrina (PR), Brasil.

5. Residente do 3o Ano do Programa de Residência Médica em Ortopedia e Traumatologia da Universidade Estadual de Londrina - UEL - Londrina (PR), Brasil.

6. Residente do 3o Ano do Programa de Residência Médica em Ortopedia e Traumatologia da Universidade Estadual de Londrina - UEL - Londrina (PR), Brasil.

Endereço para correspondência: Carlos Eduardo Sanches Vaz, Rua Borba Gato, 1.078, apto. 1.202, Jardim das Américas - 86010-630 - Londrina (PR) - Brasil. E-mail: carlos.vaz@sercomtel.com.br

Recebido em 15/1/07. Aprovado para publicação em 18/7/07.

Copyright RBO2007 ção de água destilada (grupo controle). Realizou-se uma fratura fechada médio-diafisária da tíbia e fíbula direitas em cada animal, seguida da administração diária das drogas de acordo com o grupo, durante 30 dias. Após esse período, os animais foram sacrificados para estudo dos calos ósseos formados. Os critérios de avaliação foram a avaliação clínica da consolidação óssea, mensuração da densidade mineral do calo ósseo utilizando-se a densitometria óssea e cálculo da área do calo formado por meio de planigrafia. Os dados coletados foram avaliados com a técnica da análise de variância (ANOVA) para verificar diferenças entre as médias nos quatro grupos estudados e com o teste de Tukey para comparação das médias duas a duas. Adotou-se nível de significância de $5 \%(\alpha=0,05)$. Resultados: A utilização da condroitina e da glicosamina, tanto de maneira isolada quanto associadas, não resultou em aumento da área do calo ósseo ou da sua densidade mineral óssea, e não havendo melhora clínica da consolidação óssea. Conclusão: A administração de condroitina e glicosamina, neste estudo, não influenciou - quer de maneira positiva ou negativa - a consolidação de fraturas experimentais em ratos.

Descritores - Fraturas ósseas; Consolidação da fratura/efeitos de drogas; Cartilagem articular; Condroitina; Glicosamina; Ratos

\section{ABSTRACT}

Objective: To evaluate the effects of administering chondroitin and glucosamine for bone healing in an animal model. Methods: This experimental study involved the use of 50 male adult Lewis rats. The animals were randomically 
divided into four groups of 10 animals each, as follows: group I, with administration of glucosamine; group II, with administration of chondroitin; group III, with the administration of a combination of glucosamine and chondroitin: group IV, with administration of distilled water (control group). A close fracture was produced in the mid shaft of the right tibia and of the right fibula in each animal, followed by the daily administration of the drugs according to the groups, for 30 days. After such period, the animals were sacrificed to study the bone calluses that formed. Evaluation criteria were a clinical evaluation of bone healing, measurement of the mineral density of the bone callus using bone densitometry and a planigraphic calculation of the callus area that was formed. The data collected were evaluated by the variance analysis technique (ANOVA) to check for differences among the mean values in the four groups of the study, and by the Tukey test to compare the means two by two. A significance level of $5 \%(\alpha=0,05)$ was adopted. Results: The use of chondroitin and glucosamine, either alone or in combination, did not yield an increased area of bone callus or of the bone mineral density, and there was no clinical improvement in bone healing. Conclusion: The administration of chondroitin and glucosamine in this study did not have any impact - either positive or negative on the healing of experimental fractures in rats.

Keywords - Fractures, bone; Fracture healing/drug effects; Cartilage, articular; Chondroitin; Glucosamine; Rats

\section{INTRODUÇÃO}

A consolidação óssea é um processo complexo que envolve múltiplas fases que se superpõem ${ }^{(1)}$. As respostas celulares próximas ao local da fratura levam primeiramente à produção de um tecido cartilaginoso, que posteriormente se calcifica. Esse molde cartilaginoso inicial é fundamental no reparo osso da maioria das fraturas e depende da proliferação e produção de matriz cartilaginosa não mineralizada pelos condrócitos ${ }^{(2)}$.

A glicosamina e a condroitina são substâncias atualmente empregadas no arsenal terapêutico da osteoartrose devido a seus efeitos biológicos sobre a cartilagem articular, promovendo aumento da síntese de matriz cartilaginosa, aumento da produção de ácido hialurônico pelos sinoviócitos e inibindo enzimas responsáveis pela degradação da cartilagem ${ }^{(3)}$.

O objetivo deste estudo foi avaliar um possível efeito benéfico da utilização dessas substâncias para estimular a consolidação de fraturas.

\section{MÉTODOS}

Os procedimentos descritos a seguir estão em conformidade com o Guide for the Care and Use of Laboratory Animals of International Association for Assessment and Accreditation of Laboratory Animal Care (1996) e com a Lei no 6.638 de 8 de maio de 1979, que estabelece as normas para a prática didático-científica da vivisseção de animais. O estudo foi submetido e aprovado pelo Comitê de Ética em Pesquisa Médica da Universidade Estadual de Londrina.

Para a realização do experimento foram utilizados 40 ratos Wistar do sexo masculino, adultos, com média de 18 meses de idade, que foram criados no Biotério do Hospital Universitário da Universidade Estadual de Londrina (BHU-UEL). A alimentação consistiu de ração industrial, balanceada e peletizada, e água à vontade. $\mathrm{O}$ ambiente de experimentação permaneceu iluminado com luz artificial por 12 horas contínuas diárias. Temperatura, umidade e nível de ruído foram mantidos estáveis.

Os animais foram randomicamente divididos em quatro grupos: no grupo I foram administrados diariamente, via oral, por técnica de gavagem, $2 \mathrm{ml}$ de uma solução contendo $200 \mathrm{mg}$ de sulfato de condroitina; o grupo II recebeu $2 \mathrm{ml}$ de uma solução contendo $250 \mathrm{mg}$ de sulfato de glicosamina; o grupo III recebeu $2 \mathrm{ml}$ de uma solução contendo $200 \mathrm{mg}$ de sulfato de condroitina e $250 \mathrm{mg}$ de sulfato de glicosamina; o grupo IV foi utilizado como controle e recebeu apenas $2 \mathrm{ml}$ de água destilada. Esse procedimento foi realizado diariamente durante um período de 30 dias a partir da data de realização das fraturas.

$\mathrm{O}$ animal foi previamente anestesiado por meio da administração intraperitoneal de uma solução contendo quetamina $(40 \mathrm{mg} / \mathrm{kg})$ e xilazina $(5 \mathrm{mg} / \mathrm{kg})$.

Realizou-se, então, uma fratura médio-diafisária de tíbia e fíbula no membro posterior direito, fechada, por meio de aparelho de alavanca apropriado, o que permitiu uniformização no tipo de traço fraturário produzido. A seguir, o animal foi acondicionado em caixa apropriada, aquecido e isolado dos demais, até a sua recuperação anestésica completa, quando então foi devolvido à sua gaiola no biotério. Não foi utilizado qualquer tipo de imobilização no membro fraturado e foi permitida deambulação livre.

A eutanásia foi conduzida por médico veterinário habilitado. Os animais foram sacrificados 30 dias após a data da realização das fraturas, em sala separada, longe dos outros animais. Optou-se pela eutanásia por agentes químicos, por ser menos traumática e indolor, sendo administrada uma dose intracardíaca de pentobarbital sódico $(200 \mathrm{mg} / \mathrm{kg})$. 
Os resultados obtidos foram avaliados por meio de análise da mobilidade no calo de fratura utilizando-se o teste manual de Heard, conforme descrito por Alonso et al(4), avaliação radiográfica da área do calo ósseo com planimetria e avaliação da densidade mineral do calo por meio de densitometria óssea (DEXA).

O teste de Heard( ${ }^{(4)}$ consiste de avaliação clínica conforme uma escala de 0 a 2; é atribuído grau 0 à fratura consolidada, sem mobilidade ao teste manual, grau 2 quando ocorre mobilidade franca e grau 1 ao padrão intermediário de movimentação.

Na avaliação do calo ósseo pela área radiográfica (planimetria), foram realizadas radiografias do membro posterior nas incidências ântero-posterior e perfil, com mamógrafo General Electric/Siemens ${ }^{\circledR}$ e técnica de $30 \mathrm{~mA}, 25 \mathrm{Kv}$ e $0,5 \mathrm{~s}$ de exposição (figura 1). As imagens foram digitalizadas e medida planimétrica foi determinada através mensuração bidimensional das radiografias em $\mathrm{cm}^{2}$.

Na avaliação densitométrica empregou-se um densitômetro modelo Lunar GE Medical System com varredura padrão e software próprio para pequenos animais (figura 2), resultando na medida da densidade mineral óssea dos calos ósseos em g/ $\mathrm{cm}^{2}$.

Para a análise estatística das grandezas específicas (variáveis), realizou-se estatística descritiva das amostras ordinais (quantitativas): média (M) e desvio-padrão (DP).

Os dados coletados foram avaliados com a técnica da análise de variância para verificar diferenças entre as médias nos

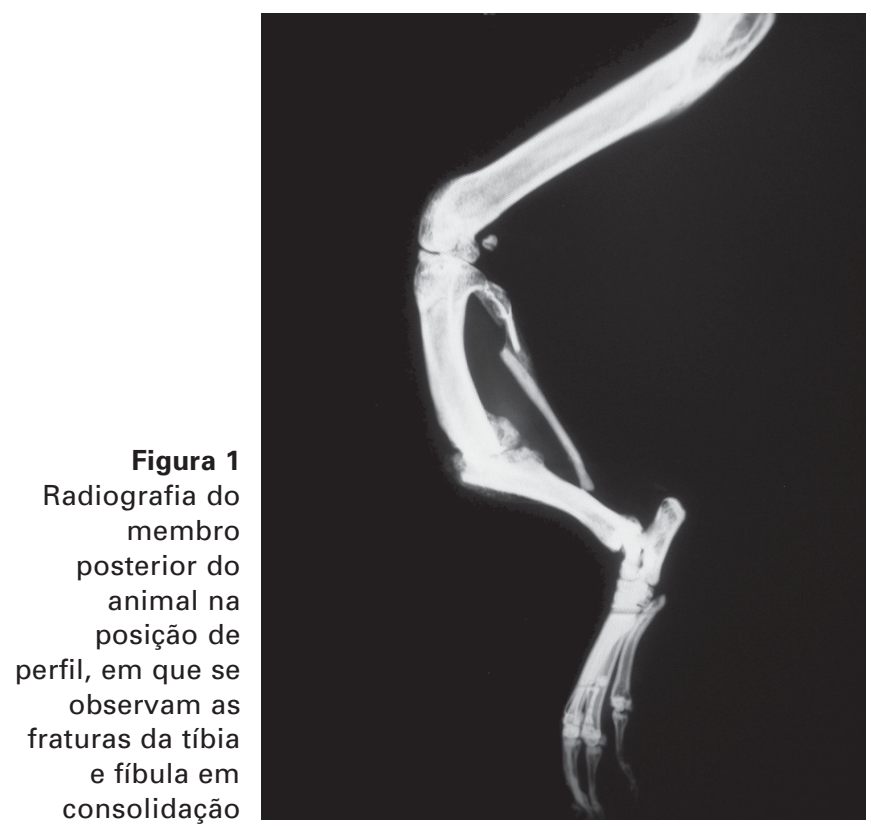

quatro grupos estudados. O teste de Tukey foi empregado para comparação das médias duas a duas. Adotou-se nível de significância de $5 \%(\alpha=0,05)$.

Utilizou-se o arredondamento científico. Nas tabelas, os valores das estatísticas descritivas, os resultados dos testes estatísticos e a probabilidade (p) foram apresentados com duas casas após a vírgula ou até o primeiro número significativo.

Utilizou-se o programa estatístico da GraphPad Software, Inc. ${ }^{\circledR}$ (1996) Graphpad Prism, versão 2.01.

\section{RESULTADOS}

A análise clínica manual dos calos ósseos, nos diferentes grupos estudados, demonstrou ausência de maior rigidez do calo nos grupos em que as drogas foram administradas, tanto de forma isolada quanto associadas, em relação ao grupo controle (tabela 1).

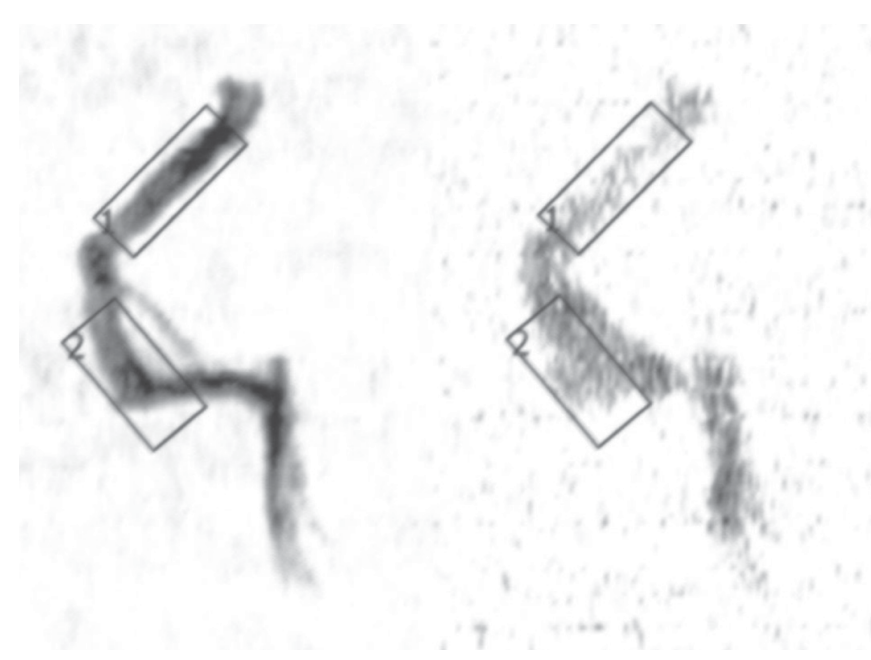

Figura 2 - Imagem de densitometria óssea do calo formado no local das fraturas no membro posterior do animal

TABELA 1

Comparação das médias das áreas dos calos ósseos formados em cada grupo estudado pela análise de variância (ANOVA) - teste de Tukey

\begin{tabular}{|c|c|c|c|c|}
\hline \multirow[t]{2}{*}{ Grupo } & \multirow[t]{2}{*}{$\mathbf{n}$} & \multicolumn{3}{|c|}{ Planimetria* } \\
\hline & & Média & $\begin{array}{l}\text { Desvio- } \\
\text { padrão }\end{array}$ & $\begin{array}{c}\text { Tukey } \\
5 \%^{+}\end{array}$ \\
\hline I (Condroitina) & 10 & 0,5110 & 0,1802 & $A$ \\
\hline II (Glicosamina) & 10 & 0,4440 & 0,2207 & A \\
\hline III (Condroitina + glicosamina) & 10 & 0,4640 & 0,1354 & $A$ \\
\hline IV Controle & 10 & 0,3210 & 0,1260 & $A$ \\
\hline
\end{tabular}

* Planimetria: teste $\mathrm{F}=2,28 \mathrm{com} 3^{\circ}$ e $36^{\circ}$ de liberdade, valor de $\mathrm{p}=0,0964$ + Médias seguidas por letras iguais não diferem pelo teste de Tukey. 
Também não ocorreram diferenças entre as médias das áreas dos calos ósseos na planigrafia (tabela 2) ou aumento da densidade mineral óssea entre os grupos estudos (tabela 3 ).

\section{DISCUSSÃO}

O aumento progressivo no número de acidentes de trânsito tem levado a grande número de vítimas com fraturas ${ }^{(5)}$, muitas vezes de difícil tratamento, principalmente quando estas são cominutivas ou se há perda de substância óssea ${ }^{(4)}$.

Apesar da expressiva evolução na abordagem inicial e no tratamento ortopédico definitivo das fraturas nos últimos anos, ainda não existem métodos seguros e livres de complicações para estimular ou acelerar a consolidação de uma fratura. Embora o emprego do enxerto ósseo autólogo seja considerado a técnica padrão, vários problemas têm sido relacionados à sua utilização(6).

Devido a isso, várias pesquisas têm sido conduzidas com o objetivo de desenvolver métodos ou medicamentos capazes de estimular a consolidação óssea. Fatores de crescimento ${ }^{(7)}$, transplante de células-tronco ${ }^{(6)}$, compostos cerâmicos ${ }^{(8-9)}$, uso

TABELA 2

\begin{tabular}{|c|c|c|c|c|}
\hline \multirow[t]{2}{*}{ Grupo } & \multirow[t]{2}{*}{$\mathbf{n}$} & \multicolumn{3}{|c|}{ BMD $^{+}$} \\
\hline & & Média & $\begin{array}{l}\text { Desvio- } \\
\text { padrão }\end{array}$ & $\begin{array}{c}\text { Tukey } \\
5 \%^{*}\end{array}$ \\
\hline Condroitina & 10 & 0,212 & 0,049 & $A$ \\
\hline Glicosamina & 10 & 0,185 & 0,039 & $A B$ \\
\hline Condroitina + glicosamina & 10 & 0,153 & 0,026 & B \\
\hline Controle & 10 & 0,211 & 0,046 & $A B$ \\
\hline
\end{tabular}

BMD: teste $F=3,38$ com $3^{\circ}$ e $36^{\circ}$ de liberdade, valor de $p=0,0587$

₹ Média seguida por letras iguais não diferem pelo teste de Tukey. de drogas que atuam no metabolismo ósseo, como os bisfosfonados $^{(10-11)}$, e aporte nutricional ${ }^{(12)}$ são algumas das opções em estudo.

Como o processo de consolidação de uma fratura depende na maioria das vezes da formação de um molde cartilaginoso inicial $^{(13)}$, realizamos este estudo empregando a condroitina e a glicosamina, drogas atualmente aceitas como capazes de estimular a síntese de matriz cartilaginosa, em uma tentativa de com isso incentivar o processo de cicatrização óssea.

O sulfato de condroitina é um açúcar complexo composto por um polímero de cadeias longas formado por unidades repetidas de dissacarídeos que, por sua vez, contêm sulfato de galactosamina e ácido glucurônico, que compõem a maioria dos glicosaminoglicanos da cartilagem articular ${ }^{(14)}$. Pesquisas demonstram que o sulfato de condroitina tem vários efeitos biológicos sobre a cartilagem, como fazer parte de um grupo de substratos disponíveis para a deposição de matriz cartilaginosa, inibir proteases e estimular a síntese de glicosaminoglicanos e colágeno ${ }^{(15-17)}$.

A glicosamina é um amino-monossacarídeo precursor da unidade de dissacarídeos dos glicosaminoglicanos, considerada esta a unidade fundamental formadora dos proteoglicanos da cartilagem articular. O mecanismo de ação da glicosamina parece envolver a inibição de proteases e a estimulação da síntese de glicosaminoglicanos; suas propriedades físicoquímicas favorecem tropismo pela cartilagem ${ }^{(14)}$.

Embora essas drogas atualmente sejam amplamente utilizadas no arsenal terapêutico da osteoartrose ${ }^{(18)}$, não encontramos na literatura estudos avaliando seu afeito na consolidação de fraturas.

O processo de consolidação de uma fratura envolve três mecanismos que interagem para a cicatrização óssea, conhecidos como ossificação endocondral, ossificação intramembranosa e formação óssea por aposição. Nas imediações do foco de fratura, as respostas celulares levam à produção de

TABELA 3

Comparação dos resultados da avaliação clínica dos calos ósseos pelo método de Heard

\begin{tabular}{|c|c|c|c|c|c|c|c|c|c|c|}
\hline \multirow[t]{2}{*}{$\begin{array}{c}\text { Grau de } \\
\text { consolidação }\end{array}$} & \multicolumn{2}{|c|}{$\begin{array}{c}\text { Condroitina + } \\
\text { glicosamina }\end{array}$} & \multicolumn{2}{|c|}{ Condroitina } & \multicolumn{2}{|c|}{ Controle } & \multicolumn{2}{|c|}{ Glicosamina } & \multicolumn{2}{|c|}{ Total } \\
\hline & $\mathbf{n}$ & $\%$ & $\mathbf{n}$ & $\%$ & $\mathbf{n}$ & $\%$ & $\mathbf{n}$ & $\%$ & $\mathbf{n}$ & $\%$ \\
\hline Alto $(0)$ & 5 & 50,0 & 5 & 50,0 & 3 & 30,0 & 5 & 50,0 & 18 & 45,0 \\
\hline Médio (1) & 3 & 30,0 & 3 & 30,0 & 5 & 50,0 & 2 & 20,0 & 13 & 32,5 \\
\hline Baixo (2) & 2 & 20,0 & 2 & 20,0 & 2 & 20,0 & 3 & 30,0 & 9 & 22,5 \\
\hline Total & 10 & 100,0 & 10 & 100,0 & 10 & 100,0 & 10 & 100,0 & 40 & 100,0 \\
\hline
\end{tabular}

$\chi^{2}=2,46$ com $6^{\circ}$ de liberdade, valor $p=0,8727$. 
tecido cartilaginoso, que posteriormente se calcifica, processo esse chamado de ossificação endocondral. Em locais mais afastados ocorre a ossificação intramembranosa diretamente, isto é, formação de osso sem necessitar de um molde cartilaginoso preexistente, apoiado em uma rede de colágeno. Finalmente, a área de ossificação por aposição direta de osso reforça o calo por inteiro ${ }^{(19)}$.

O processo de ossificação endocondral no reparo ósseo assemelha-se ao que ocorre nas placas de crescimento ósseo. Nesses locais, primeiramente, os condrócitos proliferam e produzem uma matriz cartilaginosa não mineralizada. Então, os condrócitos hipertrofiados sofrem apoptose e inicia-se a mineralização da matriz. Osteoblastos depositam osso novo nos remanescentes mineralizados da matriz cartilaginosa para produzir osso trabecular ${ }^{(20)}$. Então, a fase de remodelação se inicia, caracterizando-se por reabsorção pelos osteoclastos seguida pelo depósito de osso lamelar novo. Apesar da semelhança com as placas de crescimento, o processo de consolidação óssea é menos ordenado, com áreas de calo sofrendo remodelação entremeadas por áreas que ainda estão na fase cartilagi$\operatorname{nosa}^{(19)}$.

\section{REFERÊNCIAS}

1. Einhorn TA. The cell and molecular biology of fracture healing. Clin Orthop Relat Res. 1998;(355 Supl):S7-21.

2. McKibbin B. The biology of fracture healing in long bones. J Bone Joint Surg Br. 1978;60-B(2):150-62. Review.

3. Toffoletto O, Tavares A, Casarini DE, Redublo BM, Ribeiro AB. Farmacocinética da associação da glucosamina e sulfato de condroitina em humanos sadios do sexo masculino. Acta Ortop Bras. 2005;13(5):235-7.

4. Praemer A, Furner S, Price OP. Musculoskeletal conditions in the United States. Rosemont, IL: American Academy of Orthopedic Surgeons; 1992. p. 85-91.

5. Alonso JE, Lee J, Burgess AR, Browner BD. The management of complex orthopedic injuries. Surg Clin North Am. 1996;76(4):879-903.

6. Vaz CES. Avaliação do efeito de centrifugado osteogênico de medula óssea na consolidação de fratura: estudo experimental em coelhos [tese]. São Paulo: Faculdade de Medicina da Universidade de São Paulo; 2006.

7. Einhorn TA. Clinical applications of recombinant human BMPs: early experience and future development. J Bone Joint Surg Am. 2003;85-A Suppl 3:82-8.

8. Nade S, Armstrong L, McCartney E, Baggaley B. Osteogenesis after bone marrow transplantation. The ability of ceramic materials to sustain osteogenesis from transplanted bone marrow cells: Preliminary studies. Clin Orthop Relat Res. 1983;(181):255-63.

9. Tiedeman JJ, Garvin KL, Kile TA, Connolly JF. The role of a composite, demineralized bone matrix and bone marrow in the treatment of osseous defects. Orthopedics. 1995;18(12):1153-8.

10. Oliveira LAA, Guarniero R, Rodrigues CJ, Santana PJ, Batista MA. Avaliação do efeito do risedronato sódico na consolidação de fraturas: estudo experimental em ratos. Acta Ortop Bras. 2004;12(2):77-83.
Todos esses fatores e a freqüente dependência da consolidação óssea de uma fase cartilaginosa estimularam-nos a realizar este trabalho. Porém, os resultados encontrados neste estudo não demonstraram a eficácia das drogas estudadas na estimulação da consolidação das fraturas. Não ocorreu aumento da área do calo ósseo, melhora na densidade mineral óssea ou aumento na rigidez do calo ósseo, tanto quando as drogas foram administradas isoladamente quanto na forma de associação. Portanto, não se observou melhora quantitativa nem qualitativa nos calos ósseos formados.

\section{CONCLUSÃO}

Neste estudo, a administração de condritina e glicosamina, tanto na forma isolada quanto em associação, não influenciou - quer de maneira positiva quer negativa - o curso da consolidação de fraturas experimentais em ratos.

Os autores declaram que não existiram conflitos de interesse na realização deste trabalho.
11. Takahashi M, Yukata K, Matsui Y, Abbaspour A, Takata S, Yasui N. Bisphosphonate modulates morphological and mechanical properties in distraction osteogenesis through inhibition of bone resorption. Bone. 2006;39(3):573-81.

12. Guarniero R, Cinagava MY, Santana PJ, Batista MA, Oliveira LAA, Rodrigues CJ, Cinagava FT. Influência do componente protéico na consolidação de fraturas: trabalho experimental em ratos. Acta Ortop Bras. 2003; 11(4):206-10.

13. Brighton CT, Hunt RM. Early histological and ultrastructural changes in medullary fracture callus. J Bone Joint Surg Am. 1991;73(6):832-47. Comment in: J Bone Joint Surg Am. 1992;74(4):633-4.

14. Lippiello L. Woodward J, Karpman R, Hammad TA. In vivo chondroprotection and metabolic synergy of glucosamine and chondroitin sulfate. Clin Orthop Relat Res. 2000;(381):229-40.

15. Golding J, Ghosh P. Drugs for osteoarthrosis. II. The effects of a glycosaminoglycan polysulphate ester (Arteparon) on proteoglycan aggregation and loss from articular cartilage of immobilized rabbit knee joints. Curr Ther Res. 1983;34:67-80.

16. Hanson RR, Smalley LR, Huff GK, White S, Hamad TA. Oral treatment with a glucosamine-chondroitin sulfate compound for degenerative joint disease in horses: 25 cases. Equine Pract. 1997;19:16-22.

17. Hulth A, Lindberg L, Telhag H. Experimental osteoarthritis in rabbits. Preliminary report. Acta Orthop Scand. 1970;41(5):522-30.

18. Hungerford DS. Treating osteoarthritis with chondroprotective agents. Orthopedics Special Edition. 1998;4:39-42.

19. Greenbaum MA, Kanat, IO. Current concepts in bone healing. Review of the literature. J Am Podiatr Med Assoc. 1993;83(3):123-9.

20. Schenk RK. Biology of fracture repair. In: Browner BD, editor. Skeletal trauma: basic science, management and reconstruction. 3rd ed. Philadelphia: Saunders; 2003. v.2, p. 29-73. 